Case Report

\title{
Photobiomodulation for Complex Regional Pain Syndrome: A Case Report and Literature Summary
}

\author{
Megan Sweeney, Harold Kraft * \\ LaserMD Pain Relief, 2809 N. Sepulveda Blvd, Manhattan Beach, CA 90266, USA; E-Mails: \\ megans@lasermdpainrelief.com; hhk@lasermdpainrelief.com
}

* Correspondence: Harold Kraft; E-Mail: hhk@lasermdpainrelief.com

Academic Editor: Jih-Huah Wu

Special Issue: Photobiomodulation Therapy

OBM Integrative and Complementary Medicine 2021, volume 6 , issue 4

doi:10.21926/obm.icm.2104055
Received: September 29, 2021

Accepted: December 15, 2021

Published: December 17, 2021

\begin{abstract}
Complex Regional Pain Syndrome (CRPS) is a highly debilitating chronic pain condition characterized by continuous regional pain disproportionate to duration and intensity of the initial damaging stimulus. In CRPS, abnormal perception, transmission, and modulation of pain by nerve cells and the autonomic nervous system induces extreme increases in perceived pain that may be accompanied by swelling, temperature sensitivity, color fluctuations, and/or skin changes. CRPS most commonly affects the extremities but may spread to other limbs or bodily locations. Since there is currently no cure for the underlying processes that play a role in causing this debilitating condition, therapeutic options seek to mitigate some of the symptoms of CRPS to improve quality of life. A recent systematic review found low-quality evidence of efficacy for all currently available CRPS treatments as well as a lack of consensus about first-line treatments. The pathophysiological complexity of CRPS, in addition to the lack of non-invasive, efficacious therapies for this extremely burdensome pain condition, highlights the vital need to explore novel therapeutic options in both research and clinical practice. Photobiomodulation through the repeated application of high-intensity laser therapy in a clinical setting has been shown to modulate nociception, minimize inflammation, and reduce different types of chronic and acute pain. The present case report describes a
\end{abstract}

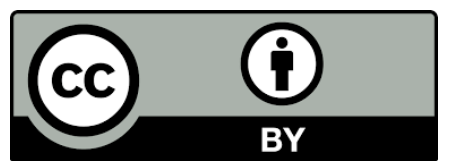

(C) 2021 by the author. This is an open access article distributed under the conditions of the Creative Commons by Attribution License, which permits unrestricted use, distribution, and reproduction in any medium or format, provided the original work is correctly cited. 
clinical protocol using laser therapy for CRPS, presents longitudinal pain scores, and summarizes mechanisms of action supporting the subsequent reduction of CRPS symptoms. After a series of laser treatments, the patient showed marked improvement in range of movement, hypersensitivity, pain, and function. Photobiomodulation through high-intensity laser therapy may represent a promising therapy for individuals affected by CRPS, although further randomized, controlled trials are recommended to evaluate efficacy on different disease-affected areas, assess dose-response relationships, and refine optimal treatment frequency.

\section{Keywords}

Photobiomodulation; complex regional pain syndrome; reflex sympathetic dystrophy; chronic pain; high intensity laser therapy

\section{Introduction}

Complex regional pain syndrome (CRPS), previously referred to as Reflex Sympathetic Dystrophy (RSD), is an uncommon and debilitating chronic pain condition marked by over 6 -months of localized spontaneous or evoked pain whose severity is disproportionate to the precipitating event [1]. Thought to result from a complex interaction between the inflammatory cascade, the central nervous system, and an impaired vasomotor response, the exact pathophysiological underpinnings driving CRPS remain uncertain. Triggering factors, clinical presentations of CRPS, and symptomatic areas vary widely, although the extremities are most frequently affected [2]. The hallmarks of CRPS often include neuropathic pain, hypersensitivity, hyperalgesia, atrophy, reduced range of motion, hair and/or nail changes, swelling, skin discoloration, and temperature intolerance - all of which severely decrease overall quality of life [3]. An interdisciplinary and multimodal approach, including pharmacological and non-pharmacological interventions, such as physical therapy, is recommended by international guidelines [4]. Nonetheless, the benefits and harms of currently recommended interventions that comprise standard of care are poorly known.

Photobiomodulation is a form of light therapy that utilizes non-ionizing forms of light sources, including lasers, LEDs, and broadband light, in the visible and infrared spectrum [5]. Light in the near-infrared range has been shown to stimulate vascular and metabolic function in a variety of cells to promote photochemical events at various biological scales [6]. This process has demonstrated positive therapeutic outcomes including but not limited to the alleviation of pain or inflammation, immunomodulation, and promotion of wound healing and tissue regeneration [7].

A subtype of Photobiomodulation, laser therapy has been used for nearly 50 years to enhance tissue healing and to relieve pain, inflammation and swelling [7, 8]. Although highly specific mechanisms of action are not entirely known, laser therapy works by a process in which the photons are absorbed in cytochrome(c) oxidase (unit four in the mitochondrial respiratory chain), and this enzyme activation increases electron transport, respiration, oxygen consumption and ATP production. A complex signaling cascade is initiated leading to activation of transcription factors and up-and-down-regulation of numerous genes [9]. Photobiomodulation Laser Therapy has been proven to achieve pain relief over the local perceived affected area [8]. Repeated clinical application 
over the central nervous system can modulate the transmission and modulation of the afferent and efferent nerve impulse transmission.

\section{Review of High Intensity Laser Therapy (HILT) for CRPS}

In CRPS, abnormal nerve cell pain perception-transmission and modulation develops over time, resulting in excess perceived pain from one or more body locations. Therapeutic options seek to ease some of the symptoms of CRPS and help to improve quality of life of individuals. The syndrome is characterized by continuous regional pain, which is disproportionate according to duration and intensity, depending on the type of the initial damage [1]. The treatment of CRPS is challenging for most pain management clinicians. Although stellate ganglion block (SGB) is often regarded as a standard treatment for CRPS, there are a wide variety of other suggested therapies including: Central Activation (spinal cord electrical stimulation, transcutaneous magnetic brain stimulation); Central Reduction in Activity (narcotics, acetaminophen, salicylates, anti-inflammatory drugs, epidural local anesthetics, ketamine, gabapentin and other anticonvulsants, antidepressants, muscle relaxants, intrathecal baclofen, cognitive behavioral therapy); Peripheral Activation (capsaicin, physiotherapy, occupational therapy), and Peripheral Reduction in Activity (antiinflammatory medications, local anesthetics, and other free-radical scavengers, botulinum toxin, corticosteroids, calcitonin, SGB and other parasympathetic blocks, calcium-channel blockers, intravenous sympathetic blockade, surgical sympathectomy, amputation) [1].

The application of laser therapy for chronic pain may provide a non-drug alternative for the management of chronic pain. This offers a promising pain management option that pharmacologic agents alone have failed to consistently demonstrate $[5,10]$. In addition, laser therapy is an affordable, accessible, non-invasive treatment modality that can produce clinical improvement relatively quickly without an array of side effects [6]. There are no established therapeutic protocols for the treatment of CRPS and laser therapy is one of the treatment options aimed at the permanent recovery of patients. The primary purpose of the treatment is to reduce pain and swelling, to improve the functionality of the affected limb and regain full mobility and muscle strength to improve functionality in the affected part of the extremity and achieve socialization of the patient [11]. Laser therapy has specific therapeutic effects, such as analgesic, anti-inflammatory, and renormalization of sympathetic nervous system tone, which further justifies its use in CRPS treatment [11]. Other studies have been conducted and have demonstrated the effectiveness of laser therapy in the treatment of CRPS [12]. Its effectiveness not only stands out in comparison with more traditional modalities such as electrical stimulation but is also used in other modalities and stands out in comparison with other therapies for pain prevention and side effects.

\section{Case Presentation}

A 35-year-old male patient presented to a Los Angeles-based outpatient pain management clinic with CRPS, a debilitating chronic pain condition that usually affects one or more limbs after an injury, surgery, or infection $[13,14]$. The patient suffered from recurrent dislocations, subluxations, and labral tears of his left shoulder while playing hockey. The patient underwent four shoulder surgeries from 2001 through 2013. In 2003, the patient reported severe neuropathic pain described as a severe burning sensation radiating down the entire left arm to the wrist (approximately $20 \mathrm{~cm}^{2}$ total affected area), accompanied by a mottled purple color and a cold temperature. The patient was 
diagnosed with Type 1 CRPS and severe myofascial neck pain, which was treated from 2003 through 2013. Invasive treatments included twelve Stellate Ganglion Blocks (SGB), both routine and CTguided, and 70 trigger point injections with either bupivacaine, liposomal bupivacaine, Depo-Medrol, or botulinum toxin. Other treatments included combinations of PT, OT, acupuncture, biofeedback, topical capsaicin, topical ketamine, topical cannabidiol combined with medical delta-9tetrahydrocannabinol (THC), opiates, non-steroidal anti-inflammatory drugs, gabapentin, pregabalin, duloxetine, carisoprodol, and diazepam.

The patient presented to this pain clinic in July 2016 with three years of chronic neuropathic pain, VAS 8/10. The patient's medications, taken in divided doses, included Hydrocodone 80mg, Duloxetine 240mg, Aripiprazole 5mg, Gabapentin 3200mg, Naproxen 1000mg, Temazepam 30mg. The patient's physical activity level remained markedly reduced with a substantial negative impact on both quality of life and employment. On physical examination, the left shoulder and upper extremity showed decreased muscle mass compared to the right. The left hand was moderately mottled and colder than the right hand. There was decreased sensation to cotton balls and monofilament in the left upper extremity. Motor strength was 4+ in the left upper extremity and $5 / 5$ in other extremities. All distal extremity pulses were intact. The range of motion was decreased only in the left upper extremity on external rotation. Full range of motion was achieved on internal rotation, abduction, adduction, flexion, and extension of the left extremity.

\subsection{Method}

We began a series of treatments of HILT, which involves powerful laser therapy that produces $>40 \mathrm{~W}$ of power at longer wavelengths, allowing for deeper tissue penetration with more effective and efficient pain relief [15]. To perform the HILT, we used two FDA-approved, Class IV, semiconductive lasers delivering light in the near-infrared spectrum. The lasers included Litecure (Lightcure Laser Therapy, New Castle, DE, USA; US FDA 510(k) \#K171343) at a power of 25Watts, and wavelengths of $810 \mathrm{~nm}$ and $980 \mathrm{~nm}$ and Aspen (Aspen Laser Systems, LLC. Denver, CO, USA; US FDA 510(k) \#K142078) at a power of 60Watts and a wavelength of 980nm. Both of these Class IV laser therapy devices are up to 1200 times more powerful than traditional cold laser therapy or lowlevel laser therapy (LLLT). Due to the size of the treatment required to address the patient's CRPS, HILT was required to enable deeper penetration into the soft tissue and muscle [5]. Negative side effects and adverse events have not been reported from the use of HILT and near-infrared (NIR) light). NIR light has been widely investigated for its ability to modulate intracellular

mechanisms related to healing [16]. HILT was chosen over LILT based on previous literature and findings that demonstrated optimal effectiveness at $80-90 \%$ per $\mathrm{cm}$ when the targets were an estimated $5 \mathrm{~cm}$ below the surface. Thus, approximately 1000 -times the skin dose is required to produce an adequate treatment effect. We used this guiding evidence to inform our treatment doses.

The participant was placed in a prone position throughout the HILT sessions. Continuous power was used with a non-contact head of the Aspen laser positioned 10 centimeters $(\mathrm{cm})$ away from the patient's skin at a 90-degree angle. The CRPS-affected area included a total of $20 \mathrm{~cm}^{2}$ centralized on the patient's left chest for a fluence of $45 \mathrm{~J} / \mathrm{cm}^{2}$. The Aspen Laser (60W laser) produces an emission spot size of $7 \mathrm{~cm}^{2}$, with a $3 \mathrm{~cm}$ diameter beam, which was directed at the patient's entire affected area of $20 \mathrm{~cm}^{2}$ with an irradiance or power density of $100 \mathrm{~mW} / \mathrm{cm}^{2}$ and an energy density of 
$8.45 \mathrm{~J} / \mathrm{cm}^{2}$. The diode laser for the Aspen Laser is a GaAlAs diode bar, and the wavelength is 980nm. It features impact structure, high efficiency and long lifetime. Following a previously published paradigm by Marinus and colleagues, we took an identical approach based on this hypothetical mechanism of action of HILT on injured tissue with aberrant inflammation, vasomotor dysfunction, or maladaptive peripheral nervous system neuroplasticity [3]. Approximately $50 \%(14 \mathrm{KJ})$ of the maximum dose $(28 \mathrm{KJ})$ was delivered to a wide region of the left chest surrounding the original surgical scar and the left shoulder girdle, $40 \%$ to the upper posterior chest wall with emphasis on the trigger points in the trapezius and extending medially as far as the spinous processes of C5 - T5, and $10 \%$ to all surfaces of the left upper extremity from the shoulder girdle down to and including the distal fingertips.

Pain scores were tracked at the beginning of each visit using the Visual Analog Scale (VAS), a validated, subjective measure for chronic pain intensity in which scores are recorded on a 0-10 scale with a handwritten mark on a $10 \mathrm{~cm}$ line that represents a continuum between $0=$ no pain to $10=$ worst pain.

\subsection{Results}

Four days after the first laser treatment with 22.5 kilojoules (KJ), the patient's pain score on the VAS decreased from 8/10 on Day 1 to $5 / 10$ on Day 4. Following the second treatment, with a dose of 26KJ, the VAS pain score decreased to $2 / 10$. The first twelve treatments were administered, on average, every 1.7 days (ranging between 1- to 4-days) over a total duration of 21-days with doses ranging from 22.5-27.0KJ (mean dose $=25 \mathrm{KJ}$ ) over the 21-days of treatment. Although the dose was lowered to $18 \mathrm{KJ}$ after the initial treatment span of 21-days, the patient's CRPS symptoms continued to improve over time with subsequent HILT treatments. Thus, the interval between treatments increased, and frequency of HILT decreased, to approximately 1 treatment with a dose of 25KJ every 4-6 days.

The VAS dropped from a total initial score of $8 / 10$ pre-treatment to 0,1 , or $2 / 10$ throughout the subsequent treatment period, except for one outlier of VAS of 6/10 (See Figure 1). For treatment episodes \#13-50, the dose was lowered to $18 \mathrm{KJ}$ per treatment, while the average treatment interval rose to 7 days as shown in Figure 2. Simultaneously, the patient's medications were reduced. Following an initial test dose of $22 \mathrm{KJ}$, doses varied from 22-28KJ for treatments 1-19.

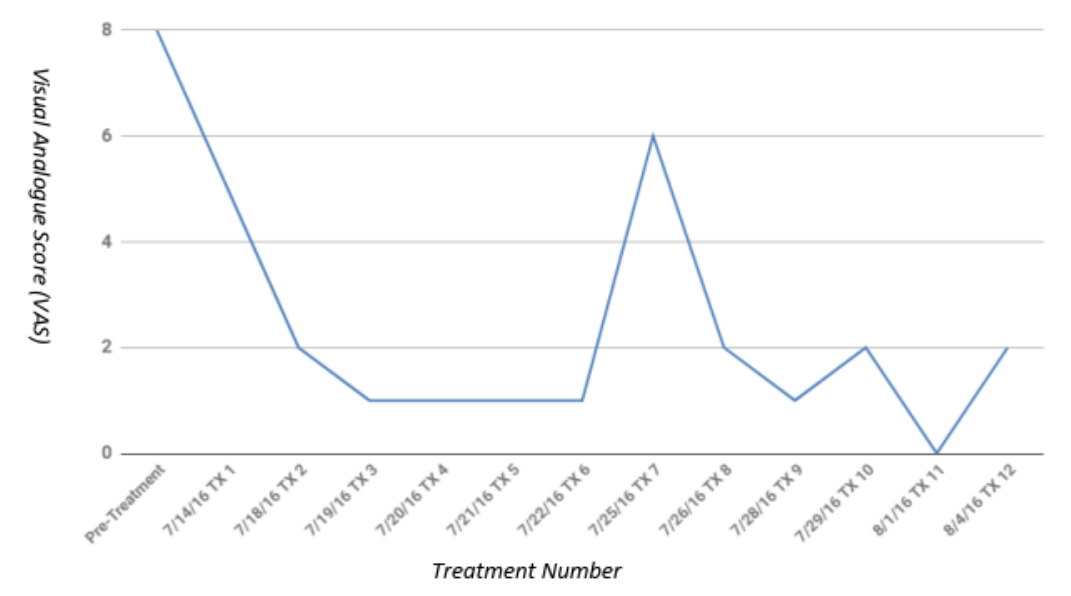

Figure 1 Visual Analogue Score (VAS) throughout the 12-treaments. 


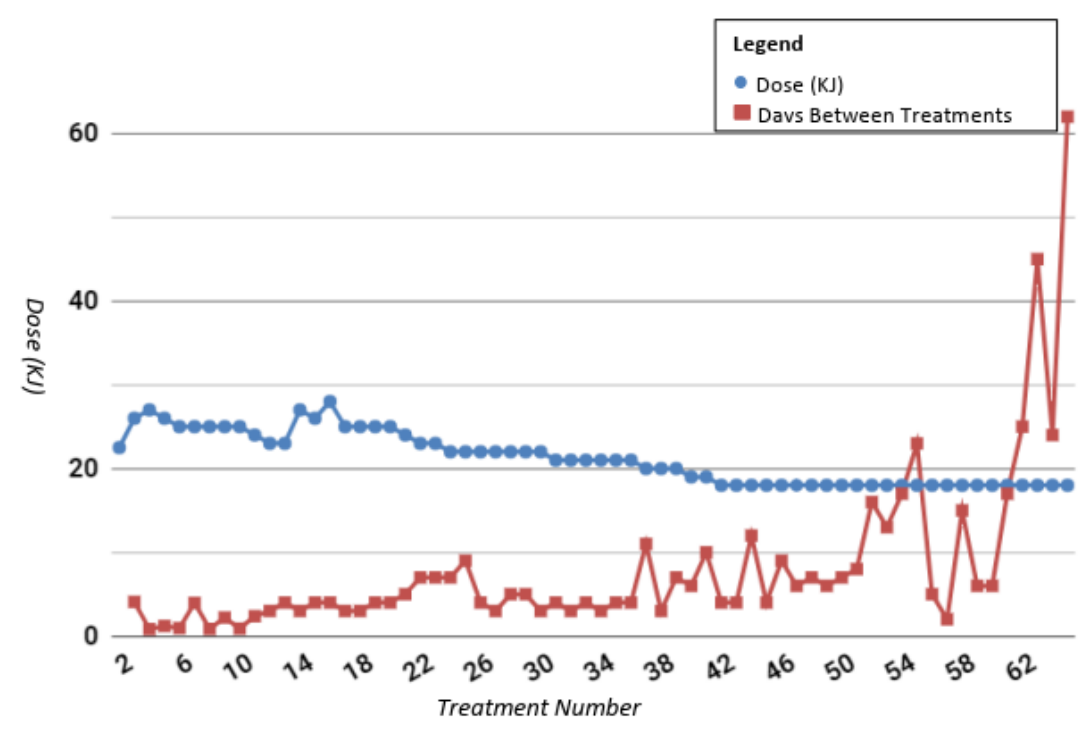

Figure 2 Laser treatment dose in kilojoules (KJ) (blue circle) and Days Between Treatments (red square), versus the treatment number.

At the end of treatment episode \#50, 32-weeks after the series of HILT treatments began, the patient had an average VAS of $0.5 / 10$. One-year post-treatment, the patient reported a VAS of $0 / 10$ and full functionality of the extremity affected by CRPS. The patient no longer requires any narcotic pain medication or gabapentin, but continues to take duloxetine, brexpiprazole, clonazepam, and cannabis as needed for sleep disturbances.

Throughout the 32-week duration comprised of 50 treatment sessions of HILT, the patient received a total of 1.4 million Joules of HILT. No side effects or adverse outcomes were reported.

\section{Discussion}

CRPS is a multifactorial disease characterized by pain, reduced function, and poor quality of life. This debilitating disease substantially contributes to global disability and has a significant economic impact. Although there are few efficacious treatment options, a multidisciplinary approach is needed for effective management of CRPS. Physical and occupational therapy, behavioral training, and medications are usually included in common clinical approaches to managing CRPS, but the evidence supporting these regimens is limited. High-intensity laser therapy involves non-invasive, FDA-approved, Class 4 medical-grade lasers that produce deeper tissue penetration and more efficacious pain relief by emitting $>40 \mathrm{~W}$ of power at longer wavelengths than low-intensity lasers. Findings of the present case study suggest that a multidisciplinary approach involving Photobiomodulation is indicated for effective management of CRPS.

Although the precise mechanisms underlying HILT and its therapeutic benefits are not fully understood, a review of the literature indicates the strongest evidence base that supports absorption of NIR photons by cytochrome coxidase in the mitochondrial respiratory chain is the key initiating event in photobiomodulation [17]. This induces an increase in cytochrome c oxidase activity which in turn increases adenosine triphosphate (ATP) production [16]. Such an increase in ATP in wounded or underperfused cells may be sufficient to activate cells in areas of injury or metabolic derangement-such as in the case of CRPS. 
HILT has been shown to be useful in nociceptive pains such as chronic back pain, osteoarthritis, post-surgical pain, fibromyalgia, and brain disorders [10, 13-15]. HILT has a potential advantage in treating CRPS in that it treats primary underlying inflammatory causes of pain. HILT also increases metabolism and blood circulation [12]. For inflammatory conditions, HILT causes the smaller arteries and lymph vessels of the human body to vasodilate, which promotes more effective clearance of inflammation, swelling, and edema from injury sites [17]. Vasodilation in lymph nodes also aides healing and restoration of homeostasis by regulating lymphatic drainage [10]. In addition, research has demonstrated that HILT decreases the pro-inflammatory response and increases the anti-inflammatory response [16].

There are no recommendations from the World Association of Laser Therapy concerning HILT laser dosage to produce optimal treatment outcomes in the setting of CRPS. To date, this case study involves a human dose of HILT higher than typically reported in the literature to treat CRPS. However, HILT has been shown to achieve greater penetration and safely produce more effective pain relief when compared to LLLT [5]. The elevated tissue temperature also promotes relief of muscle and tissue stiffness by increasing blood circulation. Prior studies have also exhibited greater clinical efficacy with higher fluence, in contrast to a bimodal model of efficacy [17]. There have been no adverse events reported in the literature after HILT [17]. Our safe and highly effective case study reinforces the safety of HILT in the setting of CRPS. Given the $N=1$ nature of a case study, limitations are inevitable. There was no control group, and the treatment approach was very personalized, with subsequent treatment doses and frequencies established based on the patient's subjective reports and pain scores. Future studies should compare HILT to other therapeutic modalities for CRPS, compare multiple treatment durations of HILT, and continue to evaluate longitudinal results to assess sustainability of HILT. Future studies with high scientific rigor are required to clarify the best treatment protocol and the long-term results.

More than 25 million Americans suffer from debilitating chronic pain conditions including CRPS. In recent decades, there has been an overreliance on the prescription of opioids for chronic pain despite their poor ability to improve function and high addiction liability. This has contributed to a significant and alarming epidemic of opioid overdose deaths compounded by unmanaged chronic pain. Innovative scientific solutions to develop alternative pain treatment options are critically needed. Additional funding devoted to Photobiomodulation can promote larger-scale trials on this promising, safe, and non-addictive form of pain management for several types of chronic pain conditions.

\section{Conclusions}

This report provides some evidence that HILT may be useful for treating CRPS, with a permanent reduction of pain in a patient with a treatment resistant CRPS. Thorough examination, prompt treatment, and a consistent therapeutic regimen resulted in a quick improvement in the patient's CRPS. Since HILT is a non-invasive, non-pharmacologic and effective therapy with few risks, administration was very well tolerated, and the patient reported no side effects or adverse events.

\section{Author Contributions}

Both authors (MS, HK) contributed equally to this clinical research project, literature review, and manuscript publication. 


\section{Competing Interests}

The authors have declared that no competing interests exist.

\section{References}

1. Rho RH, Brewer RP, Lamer TJ, Wilson PR. Complex regional pain syndrome. Mayo Clin Proc. 2002; 77: 174-180.

2. Harden RN, Bruehl S, Stanton-Hicks M, Wilson PR. Proposed new diagnostic criteria for complex regional pain syndrome. Pain Med. 2007; 8: 326-331.

3. Marinus J, Moseley GL, Birklein F, Baron R, Maihöfner C, Kingery WS, et al. Clinical features and pathophysiology of complex regional pain syndrome. Lancet Neurol. 2011; 10: 637-648.

4. Harden RN, Oaklander AL, Burton AW, Perez RS, Richardson K, Swan M, et al. Complex regional pain syndrome: Practical diagnostic and treatment guidelines. Pain Med. 2013; 14: 180-229.

5. Anders JJ, Lanzafame RJ, Arany PR. Low-level light/laser therapy versus photobiomodulation therapy. Photomed Laser Surg. 2015; 33: 183-184.

6. Hennessy M, Hamblin MR. Photobiomodulation and the brain: A new paradigm. J Opt. 2016; 19: 013003.

7. Shanks S, Leisman G. Perspective on broad-acting clinical physiological effects of photobiomodulation. In: Rehabilitation science in context. Advances in experimental medicine and biology. Cham: Springer; 2018. pp.41-52.

8. Choi HW, Lee J, Lee S, Choi J, Lee K, Kim BK, et al. Effects of high intensity laser therapy on pain and function of patients with chronic back pain. J Phys Ther Sci. 2017; 29: 1079-1081.

9. Wang X, Tian F, Soni SS, Gonzalez-Lima F, Liu H. Interplay between up-regulation of cytochromec-oxidase and hemoglobin oxygenation induced by near-infrared laser. Sci Rep. 2016; 6: 30540.

10. Rojas JC, Gonzalez-Lima F. Neurological and psychological applications of transcranial lasers and LEDs. Biochem Pharmacol. 2013; 86: 447-457.

11. Smart KM, Wand BM, O'Connell NE. Physiotherapy for pain and disability in adults with complex regional pain syndrome (CRPS) types I and II. Cochrane Database Syst Rev. 2016; 2016: CD010853.

12. Santamato A, Solfrizzi V, Panza F, Tondi G, Frisardi V, Leggin BG, et al. Short-term effects of highintensity laser therapy versus ultrasound therapy in the treatment of people with subacromial impingement syndrome: A randomized clinical trial. Phys Ther. 2009; 89: 643-652.

13. Panton L, Simonavice E, Williams K, Mojock C, Kim JS, Kingsley JD, et al. Effects of class IV laser therapy on fibromyalgia impact and function in women with fibromyalgia. J Altern Complement Med. 2013; 19: 445-452.

14. White PF, Cao X, Elvir Lazo L, Hernandez H. Effect of high-intensity laser treatments on chronic pain related to osteoarthritis in former professional athletes: A case series. J Mol Biomark Diagn. 2017; 8: 343.

15. Mohamed AM, Atya AM, Ebrahim AM, Shousha TM. Correction to: Long-term effect of highintensity laser therapy in the treatment of patients with chronic low back pain: A randomized blinded placebo-controlled trial. Lasers Med Sci. 2020; 35: 297.

16. Hamblin MR. Mechanisms and applications of the anti-inflammatory effects of photobiomodulation. AIMS Biophys. 2017; 4: 337-361. 
17. Morries LD, Cassano $P$, Henderson TA. Treatments for traumatic brain injury with emphasis on transcranial near-infrared laser phototherapy. Neuropsychiatr Dis Treat. 2015; 11: 2159-2175.

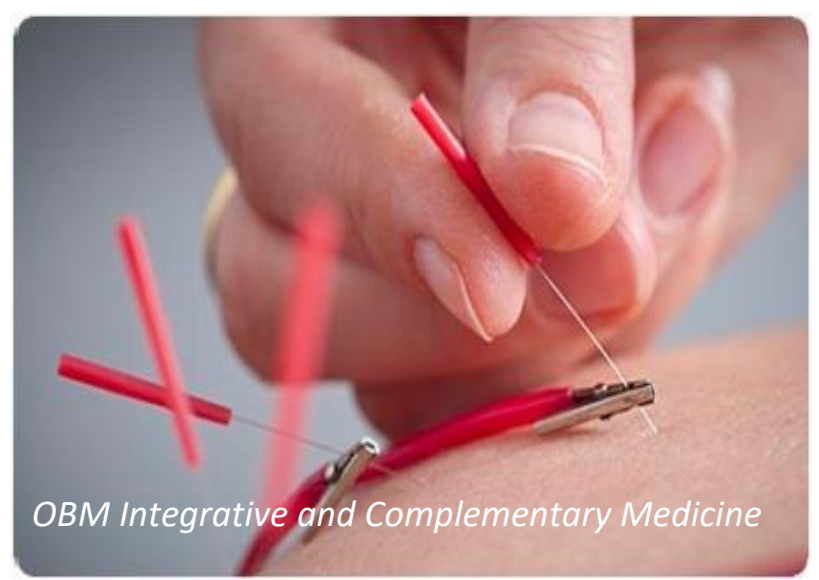

Enjoy OBM Integrative and Complementary Medicine by:

1. Submitting a manuscript

2. Joining in volunteer reviewer bank

3. Joining Editorial Board

4. Guest editing a special issue

For more details, please visit:

http://www.lidsen.com/journals/icm 